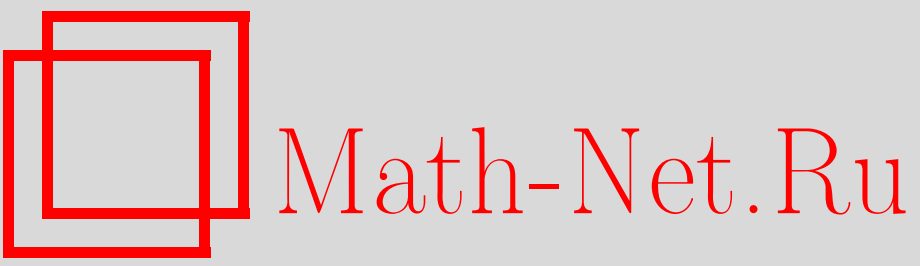

А. И. Никишов, Эквивалентные наборы решений уравнения Клейна-Гордона в постоянном электрическом поле, ТМ $\Phi$, 2003, том 136, номер 1, 77-89

DOI: https://doi.org/10.4213/tmf211

Использование Общероссийского математического портала Math-Net.Ru подразумевает, что вы прочитали и согласны с пользовательским соглашением

http://www.mathnet.ru/rus/agreement

Параметры загрузки:

IP: 35.173 .219 .12

26 апреля 2023 г., 16:48:28 
ТЕОРЕТИЧЕСКАЯ

И МАТЕМАТИЧЕСКАЯ

ФИЗИКА

Том 136, № 1

июль, 2003

(C) 2003 г.

А.И. Никишов*

\section{ЭКВИВАЛЕНТНЫЕ НАБОРЫ РЕШЕНИЙ УРАВНЕНИЯ КЛЕЙНА-ГОР ДОНА В ПОСТОЯННОМ ЭЛЕКТРИЧЕСКОМ ПОЛЕ}

Приведена развернутая аргументация в пользу выбора in- и out-состояний (среди решений волнового уравнения с одномерным потенциалом), принятого автором ранее. В этой связи изучены нестационарные и "стационарные" семейства полных наборов решений уравнения Клейна-Гордона в постоянном электрическом поле. Нестационарный набор $\psi_{p_{v}}$ состоит из решений с квантовым числом $p_{v}=p^{0} v-p_{3}$. Его можно получить из нестационарного набора $\psi_{p_{3}}$ с квантовым числом $p_{3}$ с помощью буста вдоль оси $x_{3}$ (вдоль направления электрического поля) со скоростью $-v$. Изменением калибровки решения из всех наборов приводятся к одному и тому же потенциалу без изменения квантовых чисел. При этом преобразования решений одного набора (с квантовым числом $p_{v}$ ) в решения другого набора (с квантовым числом $p_{v^{\prime}}$ ) обладают групповыми свойствами. "Стационарные" решения и наборы имеют те же свойства, что и нестационарные, и могут быть получены из стационарных решений с квантовым числом $p^{0}$ с помощью того же самого буста. Оказывается, что каждый набор можно получить из любого другого набора с помощью калибровочного преобразования. Таким образом, все наборы эквивалентны, и классификация (т.е. приписывание знака частоты и индексов in и out) в каждом наборе задается классификацией в наборе $\psi_{p_{3}}$, которая очевидна.

Ключевые слова: калибровочная инвариантность, уравнение Клейна-Гордона, полные наборы решений волнового уравнения.

\section{1. ВВЕДЕНИЕ И ПОСТАНОВКА ЗАДАЧИ}

В работах [1]-[5] рассматривались проблемы, связанные с парадоксом Клейна и рождением пар внешним полем. Выбор in- и out-решений уравнений Клейна-Гордона и Дирака с одномерным потенциалом, принятый в [1], [2] (см. также [3]), не согласуется с соответствуюшим выбором в работах [4], [5]. Таким образом, имеется проблема классификации в различных наборах решений. Мы показываем, что выбор калибровки потенциала, описываюшего постоянное электрическоеполе, фиксирует естественное квантовое число для этой калибровки. Поэтому каждая калибровка определяет полный набор решений. При этом мы можем перейти к любой другой калибровке без изменения

\footnotetext{
* Физический институт им. П. Н. Лебедева РАН, Москва, Россия. E-mail: nikishov@lpi.ru
} 
квантового числа. Таким образом, можно соотнести друг с другом решения из разных наборов, характеризуюшихся разными квантовыми числами. В то же время изменение калибровки не меняет ни физической системы, ни классификации в наборах решений. Эти простые соображения открывают путь к решению поставленной задачи.

Наша цель - показать, что классификация в наборе $\psi_{p^{0}}$ (где возникают расхождения) диктуется классификацией в наборе $\psi_{p_{3}}$, которая очевидна. В общих чертах это делается следуюшим образом. Сначала мы показываем, что набор $\psi_{p_{v}}$ может быть получен из $\psi_{p_{3}}$ путем непрерывного изменения параметра $v$ потенциала (15) (см. ниже); $v=0 \quad(v=1)$ соответствует $\psi_{p_{3}}\left(\psi_{p^{-}}\right)$. Так мы находим классификацию $\psi_{p^{-}}$. Затем мы сходным образом соотносим классификацию $\psi_{p^{0}}$ с классификацией $\psi_{p^{-}}$и, далее, с классификацией $\psi_{p_{3}}$.

Уже в классической механике можно увидеть, в каком смысле фиксация калибровки определяет сохраняющуюся величину. Решения классического уравнения движения частицы в постоянном электрическом поле имеют вид

$$
\begin{aligned}
\pi^{0}(t) & =p_{3} \operatorname{sh} \varepsilon s^{\prime}+p^{0} \operatorname{ch} \varepsilon s^{\prime}, \quad \varepsilon=\frac{e E}{m} \\
\pi_{3}(t) & =p_{3} \operatorname{ch} \varepsilon s^{\prime}+p^{0} \operatorname{sh} \varepsilon s^{\prime} \\
e E t & =p_{3}\left(\operatorname{ch} \varepsilon s^{\prime}-1\right)+p^{0} \operatorname{sh} \varepsilon s^{\prime} \\
e E x_{3} & =p_{3} \operatorname{sh} \varepsilon s^{\prime}+p^{0}\left(\operatorname{ch} \varepsilon s^{\prime}-1\right)
\end{aligned}
$$

где $s^{\prime}$ - собственное время. Мы пользуемся метрикой $\eta_{\mu \nu}=\operatorname{diag}(-1,1,1,1)$. Движение в перпендикулярном полю направлении остается свободным и в данном случае нас не интересует.

Выберем сначала векторный потенциал в виде

$$
A_{\mu}=-\delta_{\mu 3} E x_{v}, \quad x_{v}=t-v x_{3}, \quad 0 \leqslant v<1 .
$$

Из (1) и (2) следует, что

$$
\pi^{0} v-\pi_{3}+e\left(v A^{0}-A_{3}\right)=p^{0} v-p_{3} \equiv p_{v}=\mathrm{const}
$$

(векторньй потенциал берется на траектории частицы). Потенциал

$$
A_{3}=-\frac{E x_{v}}{1-v^{2}}, \quad A^{0}=-\frac{v E x_{v}}{1-v^{2}}
$$

дает ту же самую сохраняющуюся величину. Этот потенциал получается из (2) при $v=0$ с помощью буста; электрическое поле остается неизменным.

Аналогично для потенциала

$$
A^{0}=-E x_{s}, \quad x_{s}=x_{3}-s t, \quad 0 \leqslant s<1,
$$

находим, что

$$
\pi^{0}-s \pi_{3}+e\left(A^{0}-s A_{3}\right)=p^{0}-s p_{3} \equiv p_{s}=\text { const. }
$$


То же верно для потенциала

$$
A_{3}=-\frac{s E x_{s}}{1-s^{2}}, \quad A^{0}=-\frac{E x_{s}}{1-s^{2}},
$$

который получается из (5) при $s=0$ с помошью буста вдоль $x_{3}$ со скоростью $-s$. Можно было бы по-прежнему писать $v$ вместо $s$, но мы предпочитаем пользоваться различными обозначениями в разных ситуациях.

Рассмотрим теперь уравнение Клейна-Гордона

$$
\partial_{\mu} \partial^{\mu} \psi=\left[2 i e A^{\mu} \partial_{\mu}+i e\left(\partial_{\mu} A^{\mu}\right)+e^{2} A^{2}+m^{2}\right] \psi
$$

Возьмем сначала векторный потенциал, описываюший постоянное электрическое поле, в обшем виде

$$
A^{\mu}=a^{\mu} \varphi, \quad \varphi=k \cdot x, \quad k^{\mu}=\left(k^{0}, 0,0, k_{3}\right), \quad a^{\mu}=\left(a^{0}, 0,0, a_{3}\right) .
$$

Будем искать решение уравнения (8) в виде

$$
\psi_{p}=C_{p} \exp \left\{i\left[p \cdot x-\frac{p \cdot k}{k^{2}} \varphi+\frac{e a \cdot k}{2 k^{2}} \varphi^{2}\right]\right\} v_{p}
$$

Подставляя это выражение в (8), получаем

$$
\begin{gathered}
{\left[\frac{d^{2}}{d \varphi^{2}}+\left(\frac{p \cdot k}{k^{2}}\right)^{2}+2 c_{1} \varphi+c_{2} \varphi^{2}\right] v_{p}=0} \\
c_{1}=\frac{e a \cdot p}{k^{2}}-\frac{p \cdot k e a \cdot k}{\left(k^{2}\right)^{2}}, \quad c_{2}=\left(\frac{e a \cdot k}{k^{2}}\right)^{2}-\frac{e^{2} a^{2}}{k^{2}}=\frac{e^{2} E^{2}}{\left(k^{2}\right)^{2}}
\end{gathered}
$$

При переходе к переменной

$$
\tau=-c_{2}^{1 / 4}\left(\varphi+\frac{c_{1}}{c_{2}}\right)
$$

(11) сводится к уравнению для функции параболического цилиндра [6]

$$
\left[\frac{d^{2}}{d \tau^{2}}+\tau^{2}+\tilde{\lambda}\right] v_{p}=0, \quad \tilde{\lambda}=-\lambda \operatorname{sign} k^{2}, \quad \lambda=\frac{m^{2}+p_{1}^{2}+p_{2}^{2}}{|e E|}
$$

В отличие от работ [4], [7], [8], в данной статье предполагается, что заряд скалярной частицы $e=-|e|$. Рассмотрим теперь отдельно два семейства потенциалов вида (9). 


\section{2. НЕСТАЦИОНАРНЫЕ РЕШЕНИЯ}

Конкретизируем потенциал (9), полагая

$$
\begin{gathered}
a_{\mu}=\delta_{\mu 3} a, \quad k^{\mu}=(\omega, 0,0, v \omega), \quad A_{3}=-E x_{v}, \\
a \omega=E, \quad x_{v}=t-v x_{3}, \quad k^{2}=\omega^{2}\left(v^{2}-1\right) .
\end{gathered}
$$

Сохраняющимся квантовым числом является $p_{v}=p^{0} v-p_{3}$ (ср. (3)). Из (12) и (13) вытекает, что

$$
\tau_{v}=\frac{\pi_{v}}{\sqrt{|e E|\left(1-v^{2}\right)}}, \quad \pi_{v}=p_{v}-e E x_{v}, \quad p_{v}=p^{0} v-p_{3}
$$

Для краткости мы опускаем зависимость волновой функции от $x_{1}, x_{2}$ (т.е. опускаем множитель $\left.\mathrm{e}^{i p_{1} x_{1}+i p_{2} x_{2}}\right)$. Фаза в (10) удовлетворяет соотношению

$$
p_{3} x_{3}-p^{0} t-\frac{k \cdot p}{k^{2}} \varphi+\frac{e a \cdot k}{2 k^{2}} \varphi^{2}=\frac{p_{v}\left(t v-x_{3}\right)}{1-v^{2}}-\frac{e E v x_{v}^{2}}{2\left(1-v^{2}\right)}
$$

В данном разделе мы сравниваем решения, приводя их к потенциалу

$$
\tilde{A}_{\mu}=-\delta_{\mu 3} E t=A_{\mu}+\frac{\partial \eta}{\partial x^{\mu}}
$$

С учетом (15) находим

$$
\eta=-\frac{E v x_{3}^{2}}{2}, \quad \psi_{p_{v}}(x \mid \tilde{A})=\mathrm{e}^{i e \eta} \psi_{p_{v}}(x \mid A) .
$$

Поэтому вместо (17) имеем

$$
\vartheta_{v}(x \mid \tilde{A}) \equiv \vartheta_{v}=\frac{p_{v}\left(t v-x_{3}\right)}{1-v^{2}}-\frac{e E v x_{v}^{2}}{2\left(1-v^{2}\right)}-\frac{e E v x_{3}^{2}}{2} .
$$

В целях единообразия волновая функция с квантовым числом $p_{v}$ обозначается через $\psi_{p_{v}}$. Однако следует помнить, что при $v \rightarrow 0$ эта функция переходит в $\psi_{p_{3}}$, а не в $\psi_{-p_{3}}$, как можно было бы предположить, исходя из определения $p_{v}$ в (16).

Теперь мы готовы выписать и классифицировать $\left(\psi_{p_{v}} \equiv \psi_{p_{v}}(x \mid \tilde{A})\right)$-решения. Будем ставить знак частоты (см. [4]) перед $\psi$-функцией снизу для in-решения и сверху для out-решения. In-(out-)решение имеет единственную волну указанной частоты при $t \rightarrow-\infty(t \rightarrow \infty)$. Таким образом,

$$
\begin{aligned}
& { }_{ \pm} \psi_{p_{v}}=C_{p v} \mathrm{e}^{i \vartheta_{v}} D_{ \pm i \frac{\lambda}{2}-\frac{1}{2}}\left(-\mathrm{e}^{\mp i \pi / 4} T_{v}\right), \quad{ }^{ \pm} \psi_{p_{v}}=C_{p v} \mathrm{e}^{i \vartheta_{v}} D_{\mp i \frac{\lambda}{2}-\frac{1}{2}}\left(\mathrm{e}^{ \pm i \pi / 4} T_{v}\right), \\
& T_{v}=\sqrt{2} \tau_{v}=\sqrt{\frac{2}{|e E|\left(1-v^{2}\right)}}\left(p_{v}-e E x_{v}\right), \quad C_{p v}=\left[2|e E|\left(1-v^{2}\right)\right]^{-1 / 4} \mathrm{e}^{-\pi \lambda / 8} .
\end{aligned}
$$


Функции $\pm \psi_{p_{v}}$ удовлетворяют условию нормировки

$$
\int_{-\infty}^{\infty} d x_{3 \pm} \psi_{p_{v}^{\prime}}^{*} i \frac{\overleftrightarrow{d}}{d t} \pm \psi_{p_{v}}= \pm 2 \pi \delta\left(p_{v}^{\prime}-p_{v}\right)
$$

Аналогичное соотношение имеет место для ${ }^{ \pm} \psi_{p_{v}}$. Классификация возникает из условия, что при $v \rightarrow 0$ функции $\psi_{p_{v}}$ переходят в $\psi_{p_{3}}$, классификация которых дана в работе $[4]$.

Из соотношений между функциями параболического цилиндра вытекает, что

$$
\begin{aligned}
& { }_{+} \psi_{p_{v}}=c_{1 p}{ }^{+} \psi_{p_{v}}+c_{2 p}-\psi_{p_{v}}, \\
& -\psi_{p_{v}}=c_{2 p}^{*}+\psi_{p_{v}}+c_{1 p}^{*}-\psi_{p_{v}} .
\end{aligned}
$$

Коэффициенты Боголюбова $c_{1 p}, c_{2 p}$ зависят только от $\lambda$, т.е. только от $p_{\perp}^{2}=p_{1}^{2}+p_{2}^{2}[4]$ :

$$
c_{1 p}=\sqrt{2 \pi} \Gamma^{-1}\left(\frac{1-i \lambda}{2}\right) \mathrm{e}^{-\pi(\lambda-i) / 4}, \quad c_{2 p}=\mathrm{e}^{-\pi(\lambda+i) / 2}, \quad\left|c_{1 p}\right|^{2}-\left|c_{2 p}\right|^{2}=1 .
$$

Как показано в [7], [8], $\psi_{p_{v}}$ могут быть получены из $\psi_{p_{3}}$ с помощью буста вдоль оси $x_{3}$ со скоростью $-v$ и последующего калибровочного преобразования к потенщиалу $\tilde{A}_{\mu}$ (18).

Рассмотрим теперь предельный случай $v \rightarrow 1$ и получим набор функций с квантовым числом $p^{-}=p^{0}-p_{3}$ (набор для системы “с бесконечным импульсом"). Из (16) следует, что при $v \rightarrow 1$

$$
\tau_{v} \rightarrow \infty \operatorname{sign} \pi_{v}, \quad \pi_{v} \rightarrow \pi^{-}=p^{-}-e E x^{-},
$$

и нам необходимы асимптотические разложения для функций параболического цилиндра. Эти разложения содержат множители $\mathrm{e}^{ \pm i \tau^{2} / 2}$. Например,

$$
\begin{aligned}
D_{\frac{i \lambda}{2}-\frac{1}{2}}\left[-(1-i) \tau_{v}\right] \rightarrow & \left(2 \tau_{v}^{2}\right)^{-1 / 4} \exp \left[\frac{\pi}{8}(\lambda+i)+i \frac{\lambda}{4} \ln 2+\right. \\
& \left.+\frac{i \tau_{v}^{2}}{2}+\frac{i \lambda}{2} \ln \left(-\tau_{v}\right)\right], \quad \tau_{v} \rightarrow-\infty \\
D_{\frac{i \lambda}{2}-\frac{1}{2}}\left[-(1-i) \tau_{v}\right] \rightarrow & \sqrt{2 \pi} \Gamma^{-1}\left(\frac{1-i \lambda}{2}\right)\left(2 \tau_{v}^{2}\right)^{-1 / 4} \times \\
& \times \exp \left[-\frac{\pi}{8}(\lambda-i)-i \frac{\lambda}{4} \ln 2-\frac{i \tau_{v}^{2}}{2}-\frac{i \lambda}{2} \ln \tau_{v}\right]+ \\
& +\left(2 \tau_{v}^{2}\right)^{-1 / 4} \exp \left[-\frac{3 \pi}{8}(\lambda+i)+i \frac{\lambda}{4} \ln 2+\right. \\
& \left.+\frac{i \tau_{v}^{2}}{2}+\frac{i \lambda}{2} \ln \tau_{v}\right], \quad \tau_{v} \rightarrow \infty
\end{aligned}
$$

Следовательно, асимптотические выражения для $\psi$-функций в (21) содержат множители $\mathrm{e}^{i\left(\vartheta_{v} \pm \tau_{v}^{2} / 2\right)}$. Простые вычисления дают

$$
\vartheta_{v} \pm \frac{\tau_{v}^{2}}{2}= \pm \frac{p_{v}^{2}}{2|e E|\left(1-v^{2}\right)} \pm \frac{|e E| x_{v}^{2}}{2(1 \mp v)} \pm \frac{p_{v} x^{\mp}}{1 \mp v}+\frac{|e E| v x_{3}^{2}}{2}, \quad x^{ \pm}=t \pm x_{3} .
$$


Если составить волновой пакет, пользуясь функциями с фазами (27), то в пределе $v \rightarrow 1$ дают вклад лишь функции, отвечающие нижнему знаку. Расходимость постоянного члена в правой части (27) не опасна и может быть устранена с помошью компенсируюшего члена в фазе весовой функции пакета. Поэтому в пределе $v \rightarrow 1$ члены с фазой $\left(\vartheta_{v}+\tau_{v}^{2} / 2\right)$ можно опустить.

Из (21) и (26) вытекает, что при $v \rightarrow 1$

$$
+\psi_{p_{v}}(x \mid \tilde{A}) \rightarrow \exp \left[-\frac{i \pi}{8}-\frac{i \lambda}{4} \ln \frac{2}{1-v^{2}}-\frac{i p_{v}^{2}}{2|e E|\left(1-v^{2}\right)}\right]+\psi_{p^{-}}(x \mid \tilde{A}) .
$$

Аналогичные соотношения справедливы и для других функций в (21). Другими словами, при $v \rightarrow 1 \psi$-функции в $(21)$ переходят в $\psi_{p^{-}}$с точностью до несушественного фазового множителя.

Функции $\psi_{p^{-}}$для потенциала (18) определяются следуюшим образом [5]:

$$
\begin{aligned}
{ }^{+} \psi_{p^{-}}(x \mid \tilde{A}) \equiv & +\psi_{p^{-}}=(4|e E|)^{-1 / 4} \times \\
& \times \exp \left[-i \frac{p^{-} x^{+}}{2}-i e E\left(\frac{x_{3}^{2}}{2}-\frac{\left(x^{-}\right)^{2}}{4}\right)+\nu^{*} \ln \frac{\pi^{-}}{\sqrt{|e E|}}\right]
\end{aligned}
$$

и

$$
{ }_{+} \psi_{p^{-}}=\theta\left(\pi^{-}\right) c_{1 p}{ }^{+} \psi_{p^{-}}, \quad{ }^{-} \psi_{p^{-}}=\theta\left(-\pi^{-}\right) c_{1 p-} \psi_{p^{-}}, \quad \pi^{-}=p^{-}-e E x^{-},
$$

где

$$
\theta(x)=\left\{\begin{array}{ll}
1, & x>0, \\
0, & x<0,
\end{array} \quad \nu^{*}=-\frac{i \lambda}{2}-\frac{1}{2}, \quad p^{ \pm}=p^{0} \pm p_{3}, \quad x^{ \pm}=t \pm x_{3} .\right.
$$

При $\pi^{-}<0$ в (29) следует брать $\pi^{-}=\left(-\pi^{-}\right) \mathrm{e}^{-i \pi}$. Функция $-\psi_{p^{-}}$получается из ${ }^{+} \psi_{p^{-}}$ при замене знака $\pi^{-}$под знаком логарифма:

$$
-\psi_{p^{-}}(x \mid \tilde{A})=(4|e E|)^{-1 / 4} \exp \left[-i \frac{p^{-} x^{+}}{2}-i e E\left(\frac{x_{3}^{2}}{2}-\frac{\left(x^{-}\right)^{2}}{4}\right)+\nu^{*} \ln \frac{-\pi^{-}}{\sqrt{|e E|}}\right] .
$$

При $\pi^{-}>0$ в (31) следует брать $-\pi^{-}=\pi^{-} \mathrm{e}^{-i \pi}$ (случай $e=|e|$ см. в [7], [8]).

Решения $\psi_{p^{-}}$связаны с решениями с квантовым числом $p_{3}$ интегральными преобразованиями [5]:

$$
\begin{gathered}
\varphi_{p_{3}}=\int_{-\infty}^{\infty} d p^{-} N\left(p_{3}, p^{-}\right) \psi_{p^{-}} \\
N\left(p_{3}, p^{-}\right)=(2 \pi|e E|)^{-1 / 2} \exp \left\{-i \frac{\left(p^{-}\right)^{2}+4 p^{-} p_{3}+2 p_{3}^{2}}{4 e E}\right\} .
\end{gathered}
$$

В этих двух выражениях $е$ может быть любого знака, но выражения для $\psi_{p^{-}}$и $\varphi_{p_{3}}$ зависят от знака $e$ (cp. [4], [7], [8]). Ясно, что (32) и сходные выражения ниже справедливы в любой калибровке. Функции $\varphi_{p_{3}}$ отличаются от $\psi_{p_{3}}$ лишь фазовым множителем [5]:

$$
\varphi_{p_{3}}=\exp \left[-\frac{i \lambda}{4} \ln 2+i \frac{3 \pi}{8}\right] \psi_{p_{3}} .
$$


Учитьвая условия унитарности для $N\left(p_{3}, p^{-}\right)$

$$
\begin{aligned}
& \int_{-\infty}^{\infty} d p^{-} N\left(p_{3}, p^{-}\right) N^{*}\left(p_{3}^{\prime}, p^{-}\right)=\delta\left(p_{3}-p_{3}^{\prime}\right) \\
& \int_{-\infty}^{\infty} d p_{3} N\left(p_{3}, p^{-}\right) N^{*}\left(p_{3}, p^{\prime-}\right)=\delta\left(p^{-}-p^{-}\right),
\end{aligned}
$$

легко выразить $\psi_{p^{-}}$через $\varphi_{p_{3}}$ :

$$
\psi_{p^{-}}=\int_{-\infty}^{\infty} d p_{3} N^{*}\left(p_{3}, p^{-}\right) \varphi_{p_{3}}
$$

Используя тот же буст, что и при получении $\psi_{p_{v}}$ из $\psi_{p_{3}}$, находим из (32)

$$
\begin{gathered}
\varphi_{p_{v}}=\int_{-\infty}^{\infty} d p^{-} N\left(p_{v}, p^{-}\right) \psi_{p^{-}} \\
N\left(p_{v}, p^{-}\right)=(2 \pi|e E|(1-v))^{-1 / 2} \exp \left\{-i \frac{\left[p^{-}(1+v)\right]^{2}-4 p^{-} p_{v}(1+v)+2 p_{v}^{2}}{4 e E\left(1-v^{2}\right)}\right\} .
\end{gathered}
$$

Эта "матрица" также унитарна. Поэтому обратное соотношение имеет вид

$$
\psi_{p^{-}}=\int_{-\infty}^{\infty} d p_{v} N^{*}\left(p_{v}, p^{-}\right) \varphi_{p_{v}}
$$

Сопоставляя (32) и (38), получаем

$$
\begin{aligned}
\varphi_{p_{3}} & =\int_{-\infty}^{\infty} d p_{v} N\left(p_{3}, p_{v}\right) \varphi_{p_{v}} \\
N\left(p_{3}, p_{v}\right) & =\int_{-\infty}^{\infty} d p^{-} N\left(p_{3}, p^{-}\right) N^{*}\left(p_{v}, p^{-}\right)= \\
& =(2 \pi|e E| v)^{-1 / 2} \exp \left\{i \frac{p_{3}^{2}\left(1-v^{2}\right)+2 p_{v} p_{3}\left(1-v^{2}\right)+p_{v}^{2}}{2 v e E\left(1-v^{2}\right)}-i \frac{\pi}{4}\right\}
\end{aligned}
$$

Эта матриша также унитарна и, следовательно,

$$
\varphi_{p_{v}}=\int_{-\infty}^{\infty} d p_{3} N^{*}\left(p_{3}, p_{v}\right) \varphi_{p_{3}}
$$

Функция $\varphi_{p_{3}}$ Удовлетворяют условию нормировки [4]

$$
\int_{-\infty}^{\infty} d x_{3} \pm \varphi_{p_{3}^{\prime}}^{*} i \frac{\stackrel{\leftrightarrow}{d}}{d t} \pm \varphi_{p_{3}}= \pm 2 \pi \delta\left(p_{3}^{\prime}-p_{3}\right)
$$


Аналогичное условие вьполнено для ${ }^{ \pm} \varphi_{p_{3}}$. Согласно (41) имеем

$$
\begin{aligned}
\int_{-\infty}^{\infty} d x_{3} \pm \varphi_{p_{v}^{\prime}}^{*} i \frac{\overleftrightarrow{d}}{d t} \pm \varphi_{p_{v}}= & \int_{-\infty}^{\infty} d p_{3} \int_{-\infty}^{\infty} d p_{3}^{\prime} N\left(p_{3}^{\prime}, p_{v}^{\prime}\right) N^{*}\left(p_{3}, p_{v}\right) \times \\
& \times \int_{-\infty}^{\infty} d x_{3} \pm \varphi_{p_{3}^{\prime}}^{*} i \frac{\overleftrightarrow{d}}{d t} \pm \varphi_{p_{3}} .
\end{aligned}
$$

С учетом (42) и унитарности $N\left(p_{3}, p_{v}\right)$ получаем (22).

Отметим также, что соотношение (36) может быть проверено прямым вычислением. Подставляя в него ${ }^{+} \psi_{p^{-}}$из (29) и пользуясь уравнением (3.462.3) из справочника [9], получаем

$$
{ }^{+} \varphi_{p_{v}}=\left[2|e E|\left(1-v^{2}\right)\right]^{-1 / 4} \exp \left\{-\frac{\pi \lambda}{8}+i \frac{3 \pi}{8}+i \frac{\lambda}{4} \ln \frac{1+v}{2(1-v)}+i \vartheta_{v}\right\} D_{\nu^{*}}\left[(1+i) \tau_{v}\right] .
$$

Аналогичное соотношение выполнено для других функций этого набора. Здесь $\vartheta_{v}$ то же, что и в (20). Сравнение с (21) показывает, что функции $\varphi_{p_{v}}$ отличаются от $\psi_{p_{v}}$ лишь фазовым множителем:

$$
\varphi_{p_{v}}=\exp \left[i \frac{3 \pi}{8}+i \frac{\lambda}{4} \ln \frac{1+v}{2(1-v)}\right] \psi_{p_{v}} .
$$

Как и следовало ожидать, соотношение (39) переходит в (32) при $v \rightarrow 1$. Действительно, $N\left(p_{3}, p_{v}\right)$ в (40) можно представить в виде

$$
\begin{aligned}
& N\left(p_{3}, p_{v}\right)=\widetilde{N}\left(p_{3}, p_{v}\right) \exp \left[-\frac{i \pi}{4}+\frac{i p_{v}^{2}}{2|e E|\left(1-v^{2}\right)}\right], \\
& \tilde{N}\left(p_{3}, p_{v}\right)=(2 \pi|e E| v)^{-1 / 2} \exp \left\{i \frac{p_{3}^{2}(1+v)+2 p_{v} p_{3}(1+v)+p_{v}^{2}}{2 v|e E|(1+v)}\right\} .
\end{aligned}
$$

Легко видеть, что при $v \rightarrow 1$

$$
\widetilde{N}\left(p_{3}, p_{v}\right) \rightarrow N\left(p_{3}, p^{-}\right)
$$

(см. (32)). Кроме того, из (45) и (28) вытекает, что при $v \rightarrow 1$

$$
\varphi_{p_{v}} \rightarrow \exp \left\{\frac{i \pi}{4}-\frac{i p_{v}^{2}}{2|e E|\left(1-v^{2}\right)}\right\} \psi_{p^{-}} .
$$

Таким образом, фазовый множитель в правой части первого равенства (46) и фазовый множитель в правой части (48) взаимно уничтожаются.

Сопоставляя (41) и (39), заключаем, что

$$
\begin{aligned}
\varphi_{p_{v^{\prime}}}= & \int_{-\infty}^{\infty} d p_{v} N\left(p_{v^{\prime}}, p_{v}\right) \varphi_{p_{v}} \\
N\left(p_{v^{\prime}}, p_{v}\right)= & \int_{-\infty}^{\infty} d p_{3} N^{*}\left(p_{3}, p_{v^{\prime}}\right) N\left(p_{3}, p_{v}\right)=\left(-i\left(v^{\prime}-v\right) 2 \pi|e E|\right)^{-1 / 2} \times \\
& \times \exp \left\{-i \frac{p_{v^{\prime}}^{2}\left(1-v v^{\prime}\right)}{2|e E|\left(1-v^{\prime 2}\right)\left(v^{\prime}-v\right)}-\right. \\
& \left.-i \frac{p_{v}^{2}\left(1-v v^{\prime}\right)}{2|e E|\left(1-v^{2}\right)\left(v^{\prime}-v\right)}+\frac{i p_{v^{\prime}} p_{v}}{|e E|\left(v^{\prime}-v\right)}\right\}
\end{aligned}
$$


где

$$
\sqrt{-i\left(v^{\prime}-v\right)}= \begin{cases}\mathrm{e}^{-i \pi / 4} \sqrt{v^{\prime}-v}, & v^{\prime}>v, \\ \mathrm{e}^{i \pi / 4} \sqrt{v-v^{\prime}}, & v^{\prime}<v .\end{cases}
$$

Матрица $N\left(p_{v^{\prime}}, p_{v}\right)$ эрмитова и обладает групповым свойством

$$
N\left(p_{v^{\prime \prime}}, p_{v}\right)=\int_{-\infty}^{\infty} d p_{v^{\prime}} N\left(p_{v^{\prime \prime}}, p_{v^{\prime}}\right) N\left(p_{v^{\prime}}, p_{v}\right) .
$$

Подставляя ${ }^{+} \varphi_{p_{v}}={ }^{+} \varphi_{p_{v}}(x \mid \tilde{A})$ из (44) в правую часть первого равенства (49) и пользуясь уравнением (2.11.4.7) из справочника [10], получаем левую часть первого равенства (49)

В этом разделе мы сравнивали решения уравнения Клейна-Гордона с векторным потенциалом (18). Пользуясь преобразованиями, аналогичными (18), (19), можно перейти к любому векторному потенциалу рассматриваемого поля.

\section{3. СТАЦИОНАРНЫЕ РЕШЕНИЯ}

Мы называем "стационарными" решения с квантовым числом $p_{s}=p^{0}-s p_{3}$. При $s=0$ эти решения стационарны в обычном смысле. Остальные решения получаются из них при помоши бустов. Рассмотрение в данном разделе аналогично рассмотрению в предыдушем разделе.

В потенциале (9) положим

$$
\begin{gathered}
a_{\mu}=\delta_{\mu 0} a, \quad k^{\mu}=(s \omega, 0,0, \omega), \quad k^{2}=\omega^{2}\left(1-s^{2}\right), \\
A^{0}=-A_{0}, \quad a \omega=E, \quad x_{s}=x_{3}-s t .
\end{gathered}
$$

Из (13) и (12) вытекает, что

$$
\tau_{s}=\frac{\pi_{s}}{\sqrt{|e E|\left(1-s^{2}\right)}}, \quad \pi_{s}=p_{s}-|e E| x_{s}, \quad p_{s}=p^{0}-s p_{3} .
$$

Для фазы в (10) получаем

$$
p_{3} x_{3}-p^{0} t-\frac{k \cdot p}{k^{2}} \varphi+\frac{e a \cdot k}{2 k^{2}} \varphi^{2}=-\frac{p_{s}\left(t-s x_{3}\right)}{1-s^{2}}+\frac{e E s x_{s}^{2}}{2\left(1-s^{2}\right)} .
$$

В данном разделе мы приводим решения с разными $s$ к потенциалу

$$
\mathcal{A}_{\mu}=\delta_{\mu 0} E x_{3}, \quad \mathcal{A}^{0}=-\mathcal{A}_{0} .
$$

В этой калибровке фаза (53) приобретает дополнительный член:

$$
\begin{aligned}
\vartheta_{s}(x \mid \mathcal{A}) & \equiv \vartheta_{s}=-\frac{p_{s}\left(t-s x_{3}\right)}{1-s^{2}}+\frac{e E s x_{s}^{2}}{2\left(1-s^{2}\right)}+\frac{e E s t^{2}}{2}= \\
& =-\frac{p_{s}\left(t-s x_{3}\right)}{1-s^{2}}-\frac{|e E| s}{2\left(1-s^{2}\right)}\left(x_{3}^{2}-2 s t x_{3}+t^{2}\right) .
\end{aligned}
$$


Классификация семейства наборов $\psi_{p_{s}}$ диктуется требованием того, чтобы при $s=0$ получалась классификация в наборе $\psi_{p^{0}}$ (подробнее о классификации в последнем наборе см. [5]). Таким образом,

$$
\begin{aligned}
{ }_{ \pm} \psi_{p_{s}} & =C_{p s} \mathrm{e}^{i \vartheta_{s}} D_{ \pm i \frac{\lambda}{2}-\frac{1}{2}}\left( \pm \mathrm{e}^{ \pm i \pi / 4} Z_{s}\right), \\
{ }^{ \pm} \psi_{p_{s}} & =C_{p s} \mathrm{e}^{i \vartheta_{s}} D_{\mp i \frac{\lambda}{2}-\frac{1}{2}}\left( \pm \mathrm{e}^{\mp i \pi / 4} Z_{s}\right), \\
Z_{s} & =-\sqrt{2} \tau_{s}=-\sqrt{\frac{2}{|e E|\left(1-s^{2}\right)}}\left(p_{s}-|e E| x_{s}\right), \\
C_{p s} & =\left[2|e E|\left(1-s^{2}\right)\right]^{-1 / 4} \mathrm{e}^{-3 \pi \lambda / 8} .
\end{aligned}
$$

Эти функции удовлетворяют тем же соотношениям (23) с теми же $c_{1 p}, c_{2 p}$ (см. (24)).

Учитывая, что асимптотические разложения для функций параболического цилиндра содержат множители $\mathrm{e}^{ \pm i \tau_{s}^{2} / 2}$, получаем аналогичное $(27)$ выражение

$$
\vartheta_{s} \pm \frac{\tau_{s}^{2}}{2}= \pm \frac{p_{s}^{2}}{2|e E|\left(1-s^{2}\right)} \pm \frac{|e E| x_{s}^{2}}{2(1 \pm s)}-\frac{p_{s} x^{ \pm}}{1 \pm s}-\frac{|e E| s t^{2}}{2}
$$

По причинам, упомянутым после уравнения (27), необходимо рассматривать лишь случай, отвечающий верхнему знаку. Легко проверить, что при $s \rightarrow 1$

$$
\begin{gathered}
+\psi_{p_{s}}(x \mid \mathcal{A}) \rightarrow \exp \left[-\frac{i 3 \pi}{8}-\frac{i \lambda}{4} \ln \frac{2}{1-s^{2}}+\frac{i p_{s}^{2}}{2|e E|\left(1-s^{2}\right)}\right]+\psi_{p^{-}}(x \mid \mathcal{A}), \\
+\psi_{p^{-}}(x \mid \mathcal{A})=\mathrm{e}^{i e E t x_{3}+} \psi_{p^{-}}(x \mid \tilde{A}) .
\end{gathered}
$$

Для других функций $\psi_{p_{s}}$ выполнены аналогичные соотношения. Другими словами, при $s \rightarrow 1 \psi_{p_{s}}$ переходит в $\psi_{p^{-}}$с точностью до несущественного фазового множителя. Заметим теперь, что вместо потенциала (54) можно использовать (18). Можно сказать, что набор $\psi_{p^{-}}$является связуюшим звеном между $\psi_{p_{v}}{ }^{-}$и $\psi_{p_{s}}$-семействами. Все наборы в этом объединенном (супер)семействе экспериментально не различимы. Вместо классического решения (1) мы имеем суперсемейство наборов $\left(\psi_{p_{v}}, \psi_{p_{s}}\right)$ для уравнения Клейна-Гордона.

Как и в предыдушем разделе, наряду с $\psi_{p_{s}}$ рассмотрим $\varphi_{p_{s}}($ cp. $(36),(37)$ и (45)):

$$
\begin{gathered}
\varphi_{p_{s}}=\int_{-\infty}^{\infty} d p^{-} S\left(p_{s}, p^{-}\right) \psi_{p^{-}}, \quad \psi_{p^{-}}=\int_{-\infty}^{\infty} d p_{s} S^{*}\left(p_{s}, p^{-}\right) \varphi_{p_{s}} \\
S\left(p_{s}, p^{-}\right)=(2 \pi|e E|(1-s))^{-1 / 2} \exp \left\{-i \frac{\left[p^{-}(1+s)\right]^{2}-4 p^{-} p_{s}(1+s)+2 p_{s}^{2}}{4|e E|\left(1-s^{2}\right)}\right\} \\
\varphi_{p_{s}}=\exp \left[i \frac{\pi}{8}+i \frac{\lambda}{4} \ln \frac{1+s}{2(1-s)}\right] \psi_{p_{s}}
\end{gathered}
$$

При $s=0$ из (60) находим

$$
\varphi_{p^{0}}=\int_{-\infty}^{\infty} d p^{-} S\left(p^{0}, p^{-}\right) \psi_{p^{-}}
$$


и ввиду унитарности $S\left(p^{0}, p^{-}\right)$имеем

$$
\psi_{p^{-}}=\int_{-\infty}^{\infty} d p^{0} S^{*}\left(p^{0}, p^{-}\right) \varphi_{p^{0}}
$$

Отметим, что формула (62) приведена в работах [5], а (60) можно получить из (62) при помощи буста.

Сопоставляя первое соотношение в (60) и (63), находим, что

$$
\begin{aligned}
\varphi_{p_{s}} & =\int_{-\infty}^{\infty} d p^{0} S^{*}\left(p^{0}, p_{s}\right) \varphi_{p^{0}} \\
S^{*}\left(p^{0}, p_{s}\right) & =\int_{-\infty}^{\infty} d p^{-} S\left(p_{s}, p^{-}\right) S^{*}\left(p^{0}, p^{-}\right)= \\
& =(2 \pi|e E| s)^{-1 / 2} \exp \left\{i \frac{p_{0}^{2}\left(1-s^{2}\right)-2 p_{s} p^{0}\left(1-s^{2}\right)+p_{s}^{2}}{2 s|e E|\left(1-s^{2}\right)}-i \frac{\pi}{4}\right\} .
\end{aligned}
$$

Из (61) и (58) вытекает, что при $s \rightarrow 1$

$$
\varphi_{p_{s}} \rightarrow \exp \left\{-\frac{i \pi}{4}+\frac{i p_{s}^{2}}{2|e E|\left(1-s^{2}\right)}\right\} \psi_{p^{-}}
$$

а соотношение (65) может быть представлено в виде

$$
\begin{aligned}
& S^{*}\left(p^{0}, p_{s}\right)=\widetilde{S}^{*}\left(p^{0}, p_{s}\right) \exp \left[-\frac{i \pi}{4}+\frac{i p_{s}^{2}}{2|e E|\left(1-s^{2}\right)}\right] \\
& \widetilde{S}^{*}\left(p^{0}, p_{s}\right)=(2 \pi|e E| s)^{-1 / 2} \exp \left\{i \frac{p_{0}^{2}(1+s)-2 p_{s} p^{0}(1+s)+p_{s}^{2}}{2 s|e E|(1+s)}\right\} .
\end{aligned}
$$

При этом при $s \rightarrow 1$

$$
\widetilde{S}^{*}\left(p^{0}, p_{s}\right) \rightarrow S^{*}\left(p^{0}, p^{-}\right)=(2 \pi|e E|)^{-1 / 2} \exp \left\{\frac{i\left(p^{-}\right)^{2}}{4|e E|}-\frac{i p^{0} p^{-}}{|e E|}+\frac{i p_{0}^{2}}{2|e E|}\right\}
$$

Из (66)-(68) следует, что (64) переходит в (63) при $s \rightarrow 1$.

Как показано в работах [5], $\psi_{p^{0}}$ (т.е. функции $(56)$ при $s=0$ ) ортонормированы. То же верно для $\psi_{p_{s}}$ в $(64)$ ввиду унитарности $S\left(p^{0}, p_{s}\right)$ (ср. с текстом рядом с уравнениями (42), (43)).

Соотношение, обратное (64), имеет вид

$$
\varphi_{p^{0}}=\int_{-\infty}^{\infty} d p_{s} S\left(p^{0}, p_{s}\right) \varphi_{p_{s}}
$$


Сопоставляя (64) и (69), получаем

$$
\begin{aligned}
\varphi_{p_{s^{\prime}}}= & \int_{-\infty}^{\infty} d p_{s} S\left(p_{s^{\prime}}, p_{s}\right) \varphi_{p_{s}}, \\
S\left(p_{s^{\prime}}, p_{s}\right)= & \int_{-\infty}^{\infty} d p^{0} S^{*}\left(p^{0}, p_{s^{\prime}}\right) S\left(p^{0}, p_{s}\right)= \\
= & \left(i\left(s^{\prime}-s\right) 2 \pi|e E|\right)^{-1 / 2} \exp \left\{i \frac{p_{s^{\prime}}^{2}\left(1-s s^{\prime}\right)}{2|e E|\left(1-s^{\prime 2}\right)\left(s^{\prime}-s\right)}+\right. \\
& \left.+i \frac{p_{s}^{2}\left(1-s s^{\prime}\right)}{2|e E|\left(1-s^{2}\right)\left(s^{\prime}-s\right)}-\frac{i p_{s^{\prime}} p_{s}}{|e E|\left(s^{\prime}-s\right)}\right\},
\end{aligned}
$$

где

$$
\sqrt{i\left(s^{\prime}-s\right)}= \begin{cases}\mathrm{e}^{i \pi / 4} \sqrt{s^{\prime}-s}, & s^{\prime}>s \\ \mathrm{e}^{-i \pi / 4} \sqrt{s-s^{\prime}}, & s^{\prime}<s\end{cases}
$$

Матрица $S\left(p_{s^{\prime}}, p_{s}\right)$ эрмитова и обладает групповым свойством

$$
S\left(p_{s^{\prime \prime}}, p_{s}\right)=\int_{-\infty}^{\infty} d p_{s^{\prime}} S\left(p_{s^{\prime \prime}}, p_{s^{\prime}}\right) S\left(p_{s^{\prime}}, p_{s}\right)
$$

Сопоставляя теперь первое соотношение в (60) и (38), получаем

$$
\varphi_{p_{s}}=\int_{-\infty}^{\infty} d p_{v} I\left(p_{s}, p_{v}\right) \varphi_{p_{v}}
$$

где

$$
\begin{aligned}
I\left(p_{s}, p_{v}\right)= & \int_{-\infty}^{\infty} d p^{-} S\left(p_{s}, p^{-}\right) N^{*}\left(p_{v}, p^{-}\right)=\left(\pi|e E| f_{+}\right)^{-1 / 2} \times \\
& \times \exp \left\{\frac{i f_{-}}{2|e E| f_{+}}\left[\frac{p_{s}^{2}}{1-s^{2}}-\frac{p_{v}^{2}}{1-v^{2}}\right]+\frac{2 i p_{s} p_{v}}{|e E| f_{+}}-\frac{i \pi}{4}\right\}, \\
f_{ \pm}= & (1+s)(1-v) \pm(1-s)(1+v) .
\end{aligned}
$$

Аналогично из (36) и (60) находим, что

$$
\varphi_{p_{v}}=\int_{-\infty}^{\infty} d p_{s} I^{*}\left(p_{s}, p_{v}\right) \varphi_{p_{s}}
$$

При $s=v=0$ из (72) вытекает, что

$$
\varphi_{p^{0}}=\int_{-\infty}^{\infty} d p_{3} I\left(p^{0},-p_{3}\right) \varphi_{p_{3}}, \quad I\left(p^{0},-p_{3}\right)=\frac{1}{\sqrt{2 \pi|e E|}} \exp \left(\frac{-i p^{0} p_{3}}{|e E|}-\frac{i \pi}{4}\right)
$$

что согласуется с уравнением (117) в первой из статей [5].

Подставляя в правую часть $(72)$ выражение для ${ }^{+} \varphi_{p_{v}}(x \mid \mathcal{A})=\mathrm{e}^{-i|e E| t x_{3}+} \varphi_{p_{v}}$ (где $+\varphi_{p_{v}}$ определена в $(45),(21)$ и (20)) и пользуясь формулой $(2.11 .4(7))$ из книги [10], получаем функцию ${ }^{+} \varphi_{p_{s}}(x \mid \mathcal{A})$, определенную в $(61),(56)$ и $(55)$. 


\section{4. ЗАКЛЮЧЕНИЕ}

Любой набор из семейства $\left(\psi_{p_{v}}, \psi_{p_{s}}\right)$ может быть получен из любого другого набора с помощью калибровочного преобразования. Классификация в каждом наборе диктуется классификацией в наборе $\psi_{p_{3}}$, где она не вызывает сомнений. В частности, мы доказали, что классификация в наборе $\psi_{p^{0}}$, полученная в [4] на основе ожидаемого поведения (движения) волновых пакетов, вытекает также из классификации в наборе $\psi_{p_{3}}$. Таким образом, есть уверенность в правильности классификации [4], основанной на физических соображениях и применимой к любому потенциалу и частицам любого спина.

Благодарности. Я благодарен А. В. Борисову за предложенные улучшения в рукописи. Данная работа частично поддержана РФФИ (проекты № 00-15-96566 и 02-0216944).

\section{Список литературы}

[1] A. Hansen, F. Ravndal. Physica Scripta. 1981. V. 23. P. 1036.

[2] W. Greiner, B. Müller, J. Rafelski. Quantum Electrodynamics of Strong Fields. Berlin: Springer, 1985.

[3] A. Calogeracos, N. Dombey. Contemp. Phys. 1999. V. 40. P. 313.

[4] А. И. Никишов. Тр. ФИАН. 1979. Т. 111. С. 152.

[5] A. I. Nikishov. On the theory of scalar pair production by a potential barrier. hep-th/0111137; Probl. At. Sci. Tech. 2001. № 6. P. 103.

[6] Г. Бейтмен, А. Эрдейи. Высшие трансцендентные функции. Т. 2. М.: Наука, 1974.

[7] Н. Б. Нарожсный, А. И. Никишов. ТМФ. 1976. Т. 26. № 1. С. 16.

[8] Н. Б. Нарожный, А. И. Никишов. Тр. ФИАН. 1985. Т. 168. С. 175.

[9] И. С. Градитейн, И. М. Рыљик. Таблицы интегралов, сумм, рядов и произведений. М.: Физматгиз, 1963.

[10] А. П. Прудников, Ю. А. Брычков, О. И. Маричев. Интегралы и ряды. Т. 1. М.: Наука, 1981.

Поступила в редакцию 4.II.2002 г., после доработки 27.VIII.2002 г. 